\title{
Acute caffeine and capsaicin supplementation and performance in resistance training
}

\author{
Carolina B. Simões ${ }^{1}$ (D), Paula Louise C. Gomes ${ }^{1}$ (D), Ronaldo A.D. Silva ${ }^{1}$ (D), \\ Igor C.S. Fonseca $^{1}$ (D), Mariana Fonseca ${ }^{1}$ (D), Vernon M. Cruz ${ }^{1}$ (D), Marcos D.M. Drummond ${ }^{1}$ \\ ${ }^{1}$ Universidade Federal de Minas Gerais, Escola de Educação Física, Fisioterapia e Terapia \\ Ocupacional, Departamento de Esportes, Laboratório de Nutrição e Treinamento Esportivo, Belo \\ Horizonte, MG, Brazil.
}

\author{
Associate Editor: Fernanda Bruschi Marinho Priviero (D), University of South Carolina, \\ Cardiovascular Translational Research Center, Columbia, SC, USA. E-mail: fernanda. \\ bmp@gmail.com.
}

\begin{abstract}
Aim: This study aimed to evaluate the acute supplementation effects of capsaicin, caffeine, and the combined capsaicin plus caffeine on total volume (total repetitions x weight lifted), rate of perceived exertion (RPE), and side-effects on resistance training. Methods: Eleven men $(21.5 \pm 2.1$ years, $1.75 \pm 0.08 \mathrm{~m}, 79.64 \pm 10.1 \mathrm{~kg})$, trained in resistance training (experience of $4.5 \pm 2.6$ years, weekly frequency $5 \pm 0.8$ days) were recruited. This was a crossover, randomized, double-blind study. Each volunteer went through four experimental conditions: supplemented with capsaicin (12 mg), caffeine (400 mg), capsaicin plus caffeine (12 mg and $400 \mathrm{mg}$, respectively), or placebo. After supplementation, the volunteers completed four sets of back squats until failure, at $70 \%$ of one-repetition maximum, with $90 \mathrm{~s}$ of rest interval between sets. RPE was registered at the end of each set. Volunteers were asked about the occurrence of side effects, right after exercise and $24 \mathrm{~h}$ after supplementation. Results: No significant differences were found $(\mathrm{p}>0.05)$ in total volume between placebo $(5505.2 \pm 810.7 \mathrm{~kg})$, capsaicin $(6010.0 \pm 1067.0 \mathrm{~kg})$, capsaicin plus caffeine $(5885.1 \pm 1219.3 \mathrm{~kg})$, and Caffeine $(5628.6 \pm 894.4 \mathrm{~kg})$. No significant differences were found in RPE $(\mathrm{p}>0.05)$ between the experimental conditions. The effect size of the total volume was small in capsaicin and capsaicin plus caffeine $(d=0.62$ e $d=0.47$, respectively), and trivial in the caffeine condition $(d=0.15)$. Conclusion: The supplementation failed to influence resistance training performance as well as had no effects on increasing the total volume or reducing RPE.
\end{abstract}

Keywords: sports nutrition, side-effects, dietary supplements.

\section{Introduction}

Caffeine (1,3,7-trimethylxanthine) is a substance presents in teas, coffees, energy drinks, and dietary supplements, used by most elite athletes ${ }^{1,2}$. This substance can be an ergogenic aid, improving sports performance ${ }^{2,3}$, cognition $^{4}$, aerobic resistance ${ }^{5,6}$ and muscular strength ${ }^{7,8}$. Caffeine is an antagonist of the adenosine nucleoside, blocking adenosine receptors, increasing alertness, and reducing fatigue ${ }^{4}$. Another possible mechanism responsible for improving performance is the interaction between caffeine and ryanodine receptors, present in the sarcoplasmic reticulum, which can increase the release of calcium ions in the sarcoplasm ${ }^{9}$. This can contribute to greater interaction between actin and myosin filaments, generating greater strength production ${ }^{2}$.

Previous studies have shown positive results in caffeine supplementation and resistance training for specific training protocols ${ }^{1,9}$. Salatto et al. ${ }^{10}$ found a significant positive effect of $800 \mathrm{mg}$ of caffeine supplementation in the number of maximum repetitions (NMR) at the bench press and barbell press, both at $80 \%$ of one-repetition maximum (1RM). Using similar training methods at the bench press and evaluating the effects of caffeine supplementation $\left(6 \mathrm{mg} \cdot \mathrm{kg}^{-1}\right)$ at back squat, Grgic and Mikulic ${ }^{8}$ found no difference in the rate of perceived exertion (RPE) for the bench press but found a significant reduction in RPE and pain perception for back squat. Trexler et al. ${ }^{11}$ found no statistical difference in the NMR in the leg press and the bench press, at $80 \%$ of $1 \mathrm{RM}$. Therefore, conclusions about the ergogenic capacity of caffeine in resistance training are not consensual and require further studies. In addition, adverse side-effects of caffeine supplementation are reported, such as increased heart rate, headaches, nausea, and insomnia ${ }^{4,5}$. Thus, new nutritional resources must be investigated, with potential ergogenic effects like those of caffeine, such as, for example, capsaicin ${ }^{12,13}$. 
Capsaicin (8-methyl-N-vanillyl-trans-6-nonenamide) is a natural substance found in peppers that agonizes the transient potential vanilloid receptor 1 (TRPV-1), present in skeletal muscles and other peripheral tissues ${ }^{14}$. TRPV1 activation by capsaicin increases the influx of calcium from the sarcoplasmic reticulum, which, like the possible effect of caffeine on skeletal muscle, can improve muscle strength production ${ }^{11}$. Capsaicin also has an analgesic potential since the activation and desensitization of TRPV-1 regulates nociception ${ }^{14}$, which can decrease the perception of discomfort and effort during exercise, as well as caffeine. The possible increase in strength production and the decrease in the perception of discomfort can also impact the volume of the training, which stands out as an essential training variable to maximize exerciseinduced muscle hypertrophy ${ }^{15}$. The investigation of the possible ergogenic effects of capsaicin can bring satisfactory responses to individuals who are not responsive to caffeine or sensitive to its side effects.

Freitas et $\mathrm{al}^{13}$ evaluated the effects of acute capsaicin supplementation $(12 \mathrm{mg})$ on resistance training in trained men. The results demonstrated that capsaicin reduced RPE and increased performance in the back squat, at $70 \%$ of 1RM. Such improvement was verified through the increase in the number of maximum repetitions performed and, consequently, in the total volume performed by the individuals of the experimental group. Similar results were found by Freitas et al. ${ }^{16}$, in which acute capsaicin supplementation improved the performance in resistance training, after performing a previous protocol of high-intensity interval training (HIIT). The result of this study demonstrated that capsaicin supplementation resulted in lower RPE in HIIT and improved performance in subsequent resistance training.

Capsaicin supplementation can cause side-effects, including nausea, gastrointestinal problems ${ }^{6}$. However, it is believed that these effects are related to individuals' tolerance $^{17}$. Freitas et al. ${ }^{13}$ found no gastric discomfort or burning related to supplementation of $12 \mathrm{mg}$ of Capsaicin. The absence of evidence that reports the occurrence of adverse effects for this dosage of capsaicin justifies the verification of this substance as a possible ergogenic effect.

In scientific literature and sports training, different combinations of substances with caffeine are explored, determining multi-ingredient pre-workout supplements, which can increase physical performance ${ }^{19,20}$. The possible ergogenic effect of capsaicin can be complementary and synergic to the possible stimulating caffeine effect. Since such possible effects of these substances are analogous, but through different pathways, a greater reduction in fatigue might occur ${ }^{21}$. Combined supplementation of capsaicin with caffeine can, hypothetically, prolong exercise and directly impact the volume of training in resistance training. However, no studies investigated this combination or compared the effect of these substances, as well as possible side effects.

The present study aimed to investigate the effect of acute supplementation of capsaicin, caffeine, and the combination of capsaicin with caffeine in the total volume and RPE in the resistance training, in healthy trained men, in addition to possible side effects. The hypothesis is that both capsaicin and caffeine supplementation promote an increase in total volume and reduction in RPE, and the effect of combined supplementation is greater than the isolated supplementation, without side effects in any supplementation conditions.

\section{Materials and methods}

This study utilized a crossover, randomized, doubleblind design, in which each volunteer was submitted to all experimental conditions. Subjects completed seven encounters, which consisted of presentation, familiarization, 1RM test, and four experimental interventions, respectively. The experimental trials were separated by 1 week. All trials were performed at the same time of the day. The first encounter was the project presentation. The second encounter was the familiarization session to the environment, exercises, equipment, $1 \mathrm{RM}$ test, and the questions that should be answered in the following trials. In the third encounter, the 1RM test was performed in the back squat exercise. On the following four visits, each volunteer randomly and blindly consumed caffeine (CAF), capsaicin (CAP), capsaicin plus caffeine (CAPCAF), or the placebo (PLA). Subsequently, they performed the resistance exercise protocol.

The rate of perceived exertion (RPE) was collected immediately after all sets of the exercise, as well as the heart rate (HR). The perception of side effects was recorded after the end of the exercise and $24 \mathrm{~h}$ after supplementation. Lactate was not measured as previous studies found no significant difference in lactate production with caffeine and capsaicin supplementations in resistance training $^{13,22}$.

Eleven young well-trained men $(21.5 \pm 2.1$ years, $1.75 \pm 0.08 \mathrm{~m}, 79.64 \pm 10.1 \mathrm{~kg}$ ) were recruited for the study, with experience in resistance training (experience: $4.5 \pm 2.6$ years; weekly frequency: $5 \pm 0.8$ days). In addition, the volunteers should have previous experience of performing black squat exercises and repetitions until the concentric failure. As exclusions criteria, subjects could not have a history of joint, muscle, and/or bone injuries in the lower limbs and trunk, as well as a history of gastrointestinal diseases, food allergies, and cardiovascular diseases. Furthermore, they could not be users of ergogenic substances in the last 12 months and regular controlled medications, users. The volunteers were also oriented to maintain their regular diet and activity/exercise routine in the study period. This study was approved by the Ethics 
Committee of the Federal University of Minas Gerais (CAAE: 32570820.6.0000.5149).

The volunteers were instructed to eat one hour before the experimental interventions and to replicate the food and water intake of the first experimental intervention. They were instructed not to consume chili peppers or other spicy foods as well as coffee, tea, alcohol, or energy drinks for $12 \mathrm{~h}$ prior to the assessment.

At the beginning of each trial, a food and fluid intake record were made for the day before the test. To perform nutritional calculations and determine dietary profiles, the DietBox ${ }^{\circledR}$ software (version 6.8.3, Brazil) was used. Nutritional assessment was carried out to minimize the possible influences of variations in the diet on the results of the study. If a difference of more than $5 \%$ was found in the total intake of energy and macronutrients (carbohydrates, lipids, and proteins), between the daily meals of the days and meals before the experimental sessions, the volunteer would be removed from the sample. This exclusion was not necessary.

Each participant consumed two identical capsules before performing the exercise: capsaicin $(12 \mathrm{mg})$ and placebo (starch); or caffeine (400 $\left.\mathrm{mg} \cong 5 \mathrm{mg} \cdot \mathrm{kg}^{-1}\right)$ and placebo; capsaicin and caffeine $(12 \mathrm{mg}+400 \mathrm{mg} \cong 5 \mathrm{mg}$. $\mathrm{kg}^{-1}$; respectively), or placebo and placebo (Centralfarma, Ipatinga, Brazil). The $12 \mathrm{mg}$ dose of capsaicin was adopted based on the results of the studies by Freitas et al. ${ }^{13}$ and Freitas et al. ${ }^{16}$, who found positive effects on resistance training performance from acute supplementation of this substance $\left(\cong 5 \mathrm{mg} \cdot \mathrm{kg}^{-1}\right)$. The relative caffeine dosage was adopted based on the literature, which indicates that such approximate values can be effective for increasing physical performance and safe for the volunteers $^{23}$. The fixed dose of $400 \mathrm{mg}$ was adopted because it is close to a commercial dose commonly found in caffeine supplements.

After 45 min of taking the capsules, the volunteers performed the exercise. This timing was determined because capsaicin reaches a peak of concentration $45 \mathrm{~min}$ following supplementation ${ }^{12,13,16}$. Caffeine, on the other hand, reaches peak concentrations between 30 and $75 \mathrm{~min}$ after oral ingestion ${ }^{24}$. Thus, both supplements should have their maximum effect during exercise. During the resistance training, after the end of each series, a photo was shown on a mobile phone with the RPE scale described by Cabral et al. ${ }^{25}$ and used by Freitas et al. ${ }^{13}$. The volunteer had 5 to $10 \mathrm{~s}$ to choose a value regarding their perceived exertion, which was subsequently recorded.

The HR was measured using the heart rate transmitting strap, Polar model T-31 Coded (Embu das Artes, São Paulo, Brazil) and the heart monitor watch, Polar model FT1 (Embu das Artes, São Paulo, Brazil). The increase in HR is reported as one of the main side effects of caffeine supplementation $^{23}$. Thus, HR was monitored during resistance training, being recorded at the end of each series.
The volunteers were asked about the perception of side effects related to the supplemented substances. The questions were based on previous publications on possible side effects derived from caffeine intake $e^{4,5,26}$. Studies that directly investigate possible side effects of capsaicin were not found. So, the same questions were also adopted for this substance. These are questions about seven possible side effects, which should be answered as "yes" or "no", based on the volunteer's perception. The side-effects questioned were headache, intestinal or abdominal discomfort, muscle soreness, tachycardia or heart palpitation, insomnia, increased urine output (urination), and anxiety. These questions were asked after the end of each experimental session and on the following day $(24 \mathrm{~h})$ after each supplementation, to verify if there were any side effects within $24 \mathrm{~h}$ after supplementation. Initially, the questions were asked verbally, being repeated by text message after $24 \mathrm{~h}$. It was standardized that if the volunteer did not answer the questionnaire, carried out after the end of training, or sent $24 \mathrm{~h}$ after supplementation, it would imply the absence of side effects in the respective experimental session

The 1RM test protocol was performed with similar procedures to those adopted by Drummond et al. ${ }^{27}$. In the first attempt, the volunteer started with the last weight used in familiarization. Then, the volunteer was asked to perform a maximum repetition. Subsequently, the weight to be lifted was increased, and the volunteer was asked to perform a single repetition again, after a 5 -min pause. The added weight varied from 5 to $15 \%$ according to the subjective perception of the volunteers and the evaluators. This was repeated until the volunteer was unable to perform a complete repetition, therefore, the value of 1RM was that of the previous attempt. A maximum of five attempts was allowed in the same test session, with no additional sessions required for any of the volunteers.

Initially, the volunteer performed a warm-up protocol, which consisted of 5 min walking and a subsequent set of 10 squat repetitions, at $50 \%$ of their $1 \mathrm{RM}^{13}$. After a 5 -min pause, the volunteer performed 4 sets of black squats until the concentric failure (repetitions maximum), with an intensity corresponding to $70 \%$ of $1 \mathrm{RM}$ and $90 \mathrm{~s}$ of rest between sets. Aimed better control of the exercise performance, a box with adjustable height was placed behind the volunteer (Gladius, model Plyo Box, Fortaleza, Ceará, Brazil), in order to maintain the angulation of knee flexion at approximately $90^{\circ}$ in all repetitions. The volunteers were instructed to perform complete knee extension.

For recording and later comparisons of resistance performance, the total volume (total repetitions $\mathrm{x}$ weight lifted) of experimental sessions was recorded ${ }^{15}$. The data normality was verified using the Shapiro-Wilk test. The homogeneity and sphericity of the data were verified by the Levene and Mauchly tests respectively. To compare the averages of the total volume, RPE, and HR between the experimental conditions, the ANOVA one-way test of 
repeated measures was used. To identify where the differences occurred, when relevant, Bonferroni's Post-hoc was used. The analysis of the perception of side effects was performed descriptively. The level of significance adopted was $\alpha=0.05$. To verify and classify the effect size, the same procedure of Drummond et al. ${ }^{27}$ was adopted. For the statistical analysis of the data, SPSS software version 20.0 was used.

\section{Results}

The data showed normal distribution. No significant differences were found $(\mathrm{p}>0.05)$ between the Total Volume in the PLA condition $(5505.2 \pm 810.7 \mathrm{~kg})$ and the experimental conditions CAP $(6010.0 \pm 1067.0 \mathrm{~kg})$, CAPCAF $(5885.1 \pm 1219.3 \mathrm{~kg})$ and CAF $(5628.6 \pm$ $894.4 \mathrm{~kg}$ ). None of the experimental conditions showed a statistical difference $(p>0.05)$ between them. The means of the total volumes in the four experimental conditions, and the individual results, are presented in Figure 1. The total volume effect size of the experimental conditions compared to the PLA, for trained individuals, was small in the CAP and CAPCAF ( $d=0.62$ and $d=0.47$, respectively), and trivial in the CAF condition $(\mathrm{d}=0.15)$.

The means of RPE and HR did not show statistical differences in CAP, CAPCAF and CAF compared to PLA $(p>0.05)$. There were also no statistically significant differences between RPE, and HR means of experimental conditions $(p>0.05)$. Table 1 presents the RPE and HR for each of the experimental conditions.

Regarding the perception of side effects, most volunteers answered "No" for all side effects, after exercise and concerning the $24 \mathrm{~h}$ after the supplementation. The exception was in relation to the presence of muscle soreness. Most of the volunteers answered "Yes" in CAP (63.64\%) and CAPCAF (72.73\%) after exercise and in all
Table 1 - Values of the rate of perceived exertion (RPE) and heart rate (HR) for each of the experimental conditions in eleven young welltrained men.

\begin{tabular}{lcc}
\hline Condition & RPE & HR (bpm) \\
\hline PLA & $15.43 \pm 3.04$ & $163.95 \pm 15.3$ \\
CAP & $15.41 \pm 2.9$ & $164.93 \pm 16.1$ \\
CAP/CAF & $15.7 \pm 2.9$ & $168 \pm 13.5$ \\
CAF & $15.75 \pm 3.1$ & $169.11 \pm 19.1$ \\
\hline
\end{tabular}

No statistical difference was found between the conditions. PLA $=$ placebo, $\mathrm{CAP}=$ capsaicin, $\mathrm{CAP} / \mathrm{CAF}=$ capsaicin + caffeine, $\mathrm{CAF}=$ caffeine. Data are means \pm SD.

experimental conditions in the 24-h period $(\mathrm{PLA}=72.73 \% ; \mathrm{CAP}=72.73 \% ; \mathrm{CAPCAF}=.81 .82 \%$; $\mathrm{CAF}=100 \%$ ). Tables 2 and 3 show the percentage values of the responses for each side effect in each experimental condition, $15 \mathrm{~min}$ after the session and $24 \mathrm{~h}$ after supplementation, respectively.

\section{Discussion}

To date, this was the first study that compared the effects of capsaicin and caffeine supplementation, as well as combined supplementation, on resistance training performance. It was hypothesized that, compared to placebo, there would be an increase in performance in supplemented conditions, and more expressive with combined supplementation. The results, however, did not confirm the hypothesis. There was no difference in the total volume between the experimental conditions, and RPE. The result of the average of the responses of the perception of side effects indicated that most of the volunteers did not suffer adverse effects of the supplementation. This includes the heart rate response that did not present a difference with the supplementation of caffeine and capsaicin. Temple et al. ${ }^{24}$ state that caffeine supplementation can lead to

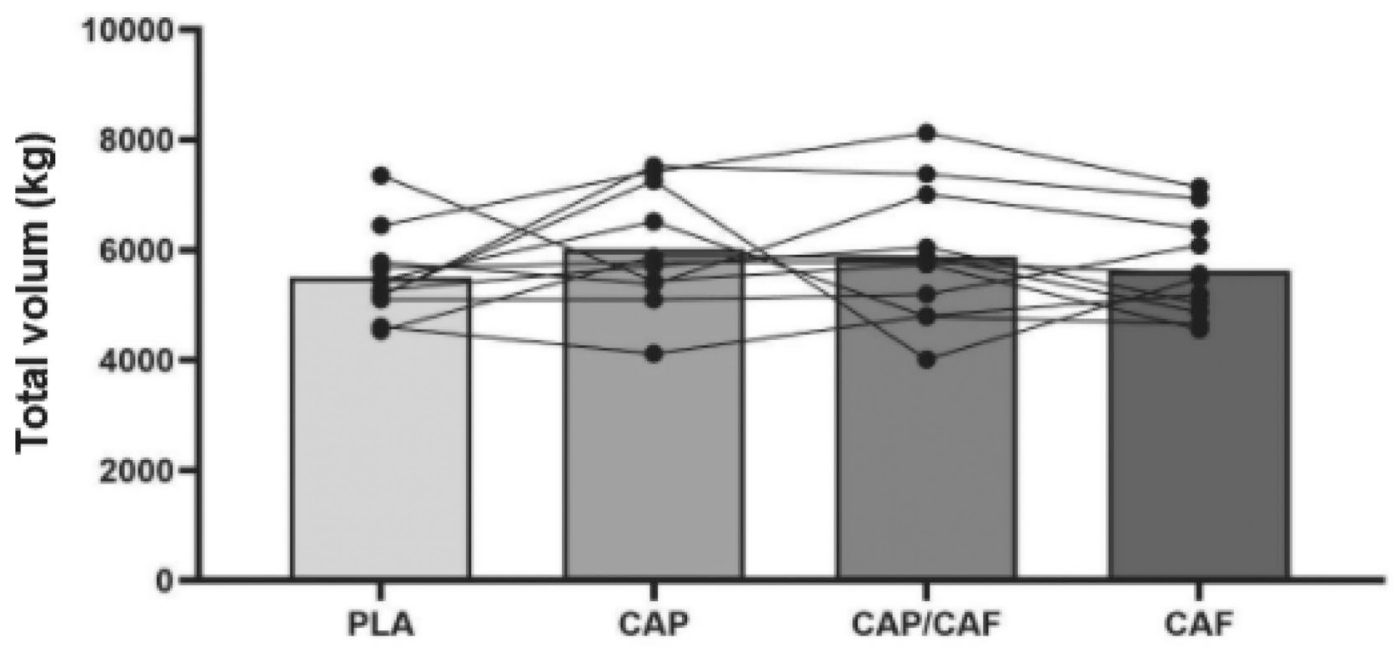

Figure 1 - Values of total volumes in the four experimental conditions in eleven young well-trained men. $\mathrm{PLA}=$ placebo, $\mathrm{CAP}=\mathrm{capsaicin}, \mathrm{CAP} / \mathrm{CAF}=$ capsaicin + caffeine, $\mathrm{CAF}=$ caffeine. Data are means $\pm \mathrm{SD}$. No statistical difference was found between the conditions. 
Table 2 - Percentage values of the responses for each side effect after the experimental condition in eleven young well-trained men.

\begin{tabular}{|c|c|c|c|c|c|c|c|c|}
\hline \multirow[b]{2}{*}{ Side-effects } & \multicolumn{2}{|c|}{ PLA } & \multicolumn{2}{|c|}{ CAP } & \multicolumn{2}{|c|}{ CAPCAF } & \multicolumn{2}{|c|}{ CAF } \\
\hline & Y (\%) & N (\%) & Y (\%) & $\mathbf{N}(\%)$ & Y (\%) & $\mathbf{N}(\%)$ & Y (\%) & $\mathbf{N}(\%)$ \\
\hline Headache & 90.09 & 90.91 & 90.09 & 90.91 & 18.18 & 81.82 & 18.18 & 81.82 \\
\hline Gastrointestinal problems & 90.09 & 90.91 & 00 & 100 & 27.27 & 72.73 & 90.09 & 90.91 \\
\hline Musclesoreness & 36.36 & 63.64 & 63.64 & 36.36 & 72.73 & 27.27 & 36.36 & 63.64 \\
\hline Tachycardia/heartpalpitations & 90.09 & 90.91 & 0 & 100 & 90.1 & 90.9 & 90.09 & 90.91 \\
\hline Insomnia & 0 & 100 & 0 & 100 & 0 & 100 & 90.09 & 90.91 \\
\hline Increased urine output & 0 & 100 & 0 & 100 & 90.1 & 90.9 & 90.09 & 90.91 \\
\hline Anxiety/nervousness & 90.09 & 90.91 & 0 & 100 & 18.18 & 81.82 & 90.09 & 90.91 \\
\hline Mean & 10.39 & 89.61 & 10.39 & 89.61 & 22.08 & 77.92 & 19.48 & 80.52 \\
\hline$\overline{\mathrm{SD}}$ & 12.23 & 12.23 & 23.72 & 23.72 & 23.97 & 23.97 & 15.24 & 15.24 \\
\hline
\end{tabular}

$\mathrm{Y}=$ Yes, $\mathrm{N}=$ No. $\mathrm{PLA}=$ placebo, $\mathrm{CAP}=$ capsaicin, $\mathrm{CAP} / \mathrm{CAF}=$ capsaicin + caffeine.

Table 3 - Percentage values of the responses for each side effect $24 \mathrm{~h}$ after supplementation in each experimental condition in leven young well-trained men.

\begin{tabular}{|c|c|c|c|c|c|c|c|c|}
\hline \multirow[b]{2}{*}{ Side-effects } & \multicolumn{2}{|c|}{ PLA } & \multicolumn{2}{|c|}{ CAP } & \multicolumn{2}{|c|}{ CAPCAF } & \multicolumn{2}{|c|}{ CAF } \\
\hline & $Y(\%)$ & $\mathbf{N}(\%)$ & Y $(\%)$ & $\mathbf{N}(\%)$ & Y (\%) & $\mathbf{N}(\%)$ & $Y(\%)$ & $\mathbf{N}(\%)$ \\
\hline Headache & 90.1 & 90.9 & 0 & 100 & 0 & 100 & 0 & 100 \\
\hline Gastrointestinal problems & 90.1 & 90.9 & 0 & 100 & 90.1 & 90.9 & 18.18 & 81.82 \\
\hline Musclesoreness & 72.73 & 27.27 & 72.73 & 27.27 & 81.8 & 18.18 & 100 & 0 \\
\hline Tachycardia/heartpalpitations & 90.09 & 90.91 & 0 & 100 & 90.1 & 90.9 & 0 & 100 \\
\hline Insomnia & 0 & 100 & 0 & 100 & 90.1 & 90.9 & 90.09 & 90.91 \\
\hline Increased urine output & 0 & 100 & 0 & 100 & 90.1 & 90.9 & 0 & 100 \\
\hline Anxiety/nervousness & 0 & 109 & 90.09 & 90,91 & 90.1 & 90.9 & 18.18 & 81.82 \\
\hline Mean & 14.29 & 85.71 & 11.69 & 88.31 & 18.18 & 81.82 & 20.78 & 79.22 \\
\hline$\overline{\mathrm{SD}}$ & 26,17 & 17.46 & 27.74 & 27.74 & 24.26 & 24.26 & 33.38 & 33.38 \\
\hline
\end{tabular}

an exaggerated increase in heart rate, which was not supported in the present study. No information about the effect of capsaicin on increasing heart rate was found in the literature. These results also indicated a low occurrence of side effects.

The total volume in the caffeine condition was not different from the other experimental conditions, with a trivial size effect, in relation to placebo. Other studies agree with the findings of the present study. Trexler et al. ${ }^{11}$ found no statistical difference in the NMR in the leg press and the bench press, at $80 \%$ of 1RM. However, Salatto et al. ${ }^{10}$ investigated the effect of $800 \mathrm{mg}$ caffeine supplementation on the volume of three sets at $80 \%$ of $1 \mathrm{RM}$ in three variations of the bench press. There was a significant increase in the NMR and, therefore, in the total volume, suggesting a possible ergogenic effect of caffeine for upper limb exercises. Thus, it can be speculated that the acute responses to caffeine are influenced by the dose, the training protocol, as well as the type of exercise used ${ }^{10}$. In addition, Brooks et al. ${ }^{19}$ concluded that there is a difference in the response to caffeine supplementation between trained and untrained men. In the respective study, untrained men showed an increase in strength, while trained men did not. As our volunteers were trained, this may be one more factor that justifies the findings.

Total volume in the capsaicin condition also showed no significant difference between the other conditions and the placebo. These results were contrary to those found by Freitas et al. ${ }^{13}$, which used the same methods as the present study. Our findings also contradict the results of other studies that investigated the effect of acute supplementation of this substance in different training protocols, such as in the $1500 \mathrm{~m}^{\text {running }}{ }^{12}$, high-intensity intermittent exercise, and subsequent resistance training ${ }^{16}$. These divergences reinforce the variation in the effect of supplementation according to the exercise protocol and individual response to supplementation. However, we do not evaluate the usual consumption of foods containing capsaicin, as well as caffeine, for analysis and discussion about the tolerance of these individuals.

The effect of combined supplementation (CAPCAF) also did not show the statistical difference, indicating that the two substances do not have a synergistic or complementary effect. However, the effect sizes of the total 
volume in the CAP (0.62) and CAPCAF condition (0.47) compared to placebo were small, not trivial. This may indicate that for some individuals there was an increase in performance from supplementation. Still, it is noted that capsaicin alone showed a result similar to the combination of the two substances, larger than caffeine effect size (trivial). This can indicate that the possible ergogenic effect for some volunteers, for this training protocol, can be due to capsaicin, not caffeine. This also suggests that there may be individuals more responsive than others and that the supplementation should not be discarded, but better investigated. In addition, the present study evaluated the effect of this supplementation in an isolated exercise. The results for a resistance training session and program, consisting of multiple exercises, may be different, due to a possible cumulative effect of fatigue throughout the training session. The lack of studies evaluating combined supplementation with these substances limits the discussion of the results. Therefore, further studies with different doses, samples, and training protocols must be carried out to determine the effect of this combined supplementation.

The RPE results showed no significant difference in any of the experimental conditions compared to placebo, or between them. However, Freitas et al. ${ }^{13}$ and Freitas et al. ${ }^{12}$ found a reduction in RPE due to acute supplementation of $12 \mathrm{mg}$ of capsaicin, in resistance training and the $1500 \mathrm{~m}$ running, respectively. The possible justification presented by these studies for the results found is that the activation of the TRPV-1 receptor-induced by capsaicin can lead to an analgesic effect and, therefore, increase the discomfort threshold during the training session. Regarding caffeine, the findings of the present study are also like those obtained by Salatto et al. ${ }^{10}$, which found no difference in RPE, between the condition caffeine and placebo, in resistance training. Grgic and Mikulic ${ }^{1}$ also found no difference in the RPE in the bench press but found a significant reduction in the RPE in the back squat, the same used in the present study. The divergences in the results indicate that a possible positive effect on RPE can be specific to the characteristic of the modality and individual, mainly. Further studies are needed to investigate such possible relations in supplementing these substances.

The result of the average of the responses of the perception of side effects indicated that the majority of the volunteers did not suffer adverse effects of the supplementation. These results confirm the safety of the doses used. The side effects of caffeine, although widely described in the literature, depending on doses and individual tolerance $^{29}$. The dose $(400 \mathrm{mg})$ was chosen both because it is a commercial dose, and it has a relative dose ergogenic potential $\left(\cong 5 \mathrm{mg} \cdot \mathrm{kg}^{-1}\right)^{3}$. Combined supplementation (CAPCAF) was expected to have a higher incidence of side effects. However, the results refuted this, because the occurrence of "No" answers after training and $24 \mathrm{~h}$ after supplementation was high $(77.92 \%$ and $81.82 \%$, respec- tively). Only the item "Muscle soreness" was reported more frequently for all experimental conditions, but it is justified by the high intensity of the exercise performed and not necessarily by the side effects of the substances. As well as the ergogenic effect, the side effects also depend on the individual's usual daily consumption ${ }^{30}$. The present study did not evaluate the intake of caffeine and capsaicin in food sources before the trials, however, the volunteers were oriented to maintain their regular diet routine during the study period. Thus, other doses should be studied in resistance training, as well as their possible side effects and the tolerance of the sample to the substance.

\section{Conclusions}

Capsaicin, caffeine, and the combined caffeine and capsaicin supplementation did not influence resistance training performance. The supplementation of these substances was not effective in increasing the total volume and reducing the RPE in resistance training. Although, the doses of the supplements were well tolerated by the volunteers since there was a low incidence of side effects. In addition, the small effect size in CAP and CAPCAF situations indicates that some individuals may benefit from supplementation with these substances.

\section{Acknowledgments}

The authors are grateful for the support of the Pró-Reitoria de Pesquisa (PRPQ) of the Federal University of Minas Gerais.

\section{References}

1. Grgic J, Grgic I, Pickering C, Schoenfeld BJ, Bishop DJ, Pedisic Z. Wake up and smell the coffee: caffeine supplementation and exercise performance-an umbrella review of 21 published meta-analyses. Br J Sports Med. 2020;54 (11):681-688. doi.

2. Southward K, Rutherfurd-Markwick KJ, Ali A. The effect of acute caffeine ingestion on endurance performance: a systematic review and meta-analysis. Int $\mathrm{J}$ Sports Med. 2018;48(8):1913-28. doi.

3. Apostolidis A, Mougios V, Smilios I, Rodosthenous J, Hadjicharalambous M. Caffeine supplementation: ergogenic in both high and low caffeine responders. Int J Sport Physiol. 2019;14(5):650-7. doi.

4. McLellan TM, Caldwell JA, Lieberman HR. A review of caffeine's effects on cognitive, physical, and occupational performance. Neurosci Biobehav Rev. 2016;71:294-312. doi.

5. Wikoff D, Welsh BT, Henderson R, Brorby GP, Britt J, Myers E, et al. Systematic review of the potential adverse effects of caffeine consumption in healthy adults, pregnant women, adolescents, and children. Food Chem Toxicol. 2017;109(1):585-648. doi. 
6. Srinivasan K. Biological activities of red pepper (Capsicum annuum) and its pungent principle capsaicin: a review Crit Rev Food Sci Nutr. 2016;56(9):1488-1500. doi.

7. Black CD, Waddell DE, Gonglach AR. Caffeine's ergogenic effects on cycling: neuromuscular and perceptual factors. Med Sci Sports Exerc. 2015;47(6):1145-58. doi.

8. Grgic J, Mikulic P. Caffeine ingestion acutely enhances muscular strength and power but not muscular endurance in resistance-trained men. Eur J Sports Sci. 2017;17(8):102936. doi.

9. Rojo-Ruiz J, Rodríguez-Prados M, Delrio-Lorenzo A, Alonso MT, García-Sancho J. Caffeine chelates calcium in the lúmen of the endoplasmic reticulum. Biochem J. 2018;475(22):3639-49. doi.

10. Salatto, RW, Arevale JA, Brown LE, Wiersma LD, Coburn JW. Caffeine's effects on an upper-body resistance exercise workout. J Strength Cond Res. 2020;34(6):1643-8. doi.

11. Trexler ET, Smith-Ryan AE, Roelofs EJ, Hirsch KR, Mock MG. Effects of coffee and caffeine anhydrous on strength and sprint performance. Eur J Sports Sci. 2016;16(6):70210. doi.

12. de Freitas MC, Cholewa JM, Gobbo LA, de Oliveira JVNS, Lira FS, Rossi FE. Acute capsaicin supplementation improves 1,500-m running time-trial performance and rate of perceived exertion in physically active adults. J Strength Cond Res. 2018;32(2):572-7. doi.

13. de Freitas MC, Cholewa JM, Freire RV, Carmo BA, Bottan $\mathrm{J}$, Bratfich $\mathrm{M}$, et al. Acute capsaicin supplementation improves resistance training performance in trained men. J Strength Cond Res. 2018;32(8):2227-32. doi.

14. Hudson AS, Kunstetter AC, Damasceno WC, Wanner SP. Involvement of the TRPV1 channel in the modulation of spontaneous locomotor activity, physical performance, and physical exercise-induced physiological responses. Braz J Med Biol Res. 2016;49(6):e5183. doi.

15. Schoenfeld BJ, Ogborn D, Krieger JW. Dose-response relationship between weekly resistance training volume and increases in muscle mass: a systematic review and metaanalysis. J Sports Sci. 2017;35(11):1073-82.

16. de Freitas MC, Cholewa JM, Panissa VLG, Toloi GG, Netto HC, Zanini de Freitas C, et al. Acute capsaicin supplementation improved resistance exercise performance performed after a high-intensity intermittent running in resistance-trained men. J Strength Cond Res. 2019 Nov 28. Epub ahead of print. doi.

17. Arnold J, Bruce-Low S, Sammut L. Oral capsaicin ingestion: a brief update - dose, tolerance, and side-effects. J Herb Med. 2016;5(2):1-5. doi.

18. Bergstrom HC, Byrd MT, Wallace BJ, Clasey JL. Examination of a multi-ingredient pre workout supplement on total volume of resistance exercise and subsequent strength and power performance. J Strength Cond Res. 2018;32(6):147990. doi.

19. Brooks JH, Wyld K, Chrismas BCR. Acute effects of caffeine on strength performance in trained and untrained individuals. J Athl Enhanc. 2015;4:6. doi.

20. Gonzalez AM, Pinzone AG, Bram J, Salisbury JL, Lee S, Mangine GT. Effect of multi-ingredient pre-workout sup- plementation on repeated sprint performance in recreationally active men and women. J Strength Cond Res. 2020;34 (4):918-23. doi.

21. Proschinger S, Freese J. Neuroimmunological and neuroenergetic aspects in exercise-induced fatigue. Exerc Immunol Rev. 2019;25:8-19. PMID: 30753129.

22. Lopes-Silva JP, da Rocha ALS, Rocha JCC, Silva VFS, Correia-Oliveira CR. Caffeine ingestion increases the upper-body intermittent dynamic strength endurance performance of combat sports athletes. Eur J Sport Sci. 2021;2:110. doi.

23. Grgic J, Trexler ET, Lazinica B, Pedisic Z. Effects of caffeine intake on muscle strength and power: a systematic review and meta-analysis. J Int Soc Sports Nutr. 2018;15:11. doi.

24. Temple JL, Bernard C, Lipshultz SE, Czachor JD, Westphal JA, Mestre MA. The safety of ingested caffeine: a comprehensive review. Front Psychiatry. 2017;8:80. doi.

25. Cabral LL, Nakamura FY, Stefanello JMF, Pessoa LCV, Smirmaul BPC, Pereira G. Initial validity and reliability of the portuguese borg rating of perceived exertion 6-20 scale. Meas Phys Educ Exerc Sci. 2020;24:103-14. doi.

26. Wilk M, Krzysztofik M, Filip A, Zajac A, Coso JD. The effects of high doses of caffeine on maximal strength and muscular endurance in athletes habituated to caffeine. Nutrients. 2019;11:1912. doi.

27. Drummond MDM, Couto BP, Oliveira MP, Szmuchrowski LA. Effects of local vibration on dynamic strength training. J Strength Cond Res. 2021;35(11):3028-34. doi.

28. Shabir A, Hooton A, Tallis J, Higgins M. The influence of caffeine expectancies on sport, exercise, and cognitive performance. Nutrients. 2018;10(10):1528. doi.

29. Vitale K, Getzin A. Nutrition and supplement update for the endurance athlete: review and recommendations. Nutrients. 2019;11(6):1289. doi.

30. Salinero JJ, Lara B, Del Coso J. Effects of acute ingestion of caffeine on team sports performance: a systematic review and meta-analysis. Res Sports Med. 2019;27(2):238-56. doi.

\section{Corresponding author}

Marcos D.M. Drummond. Universidade Federal de Minas Gerais, Escola de Educação Física, Fisioterapia e Terapia Ocupacional, Departamento de Esportes, Laboratório de Nutrição e Treinamento Esportivo, Belo Horizonte, MG, Brazil.

E-mail: zangmarcos@gmail.com.

Manuscript received on July 19, 2021

Manuscript accepted on October 6, 2021

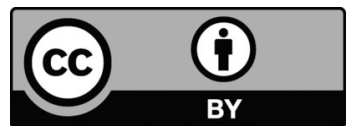

Motriz. The Journal of Physical Education. UNESP. Rio Claro, SP, Brazil - eISSN: 1980-6574 - under a license Creative Commons - Version 4.0 